Supporting Information of

\title{
Decoupling Critical Parameters in Large-range Crystallinity Controlled Polypyrrole-based High-performance Organic Electrochemical Transistors
}

Ji Hwan Kim,,+ Zubair Ahmad,,+ Youngseok Kim, ${ }^{\ddagger}$ Wonbin Kim,, Hyungju Ahn, ${ }^{\S}$ Jae-Suk Lee, $*,+$ and Myung-Han Yoon*,

\$School of Materials Science and Engineering, Gwanju Institute of Science and Technology, 123

Cheomdangwagi-ro, Buk-gu, Gwangju 61005, Republic of Korea

§Pohang Accelerator Laboratory, Pohang, Kyungsangbuk-do 37673, Republic of Korea

†These authors contributed equally to this work.

*Corresponding authors:

Prof. Jae-Suk Lee, Email: jslee@gist.ac.kr

Prof. Myung-Han Yoon, Email: mhyoon@gist.ac.kr

This file includes:

Figures S1 to S7

SI References 


\section{Supplementary Figures}
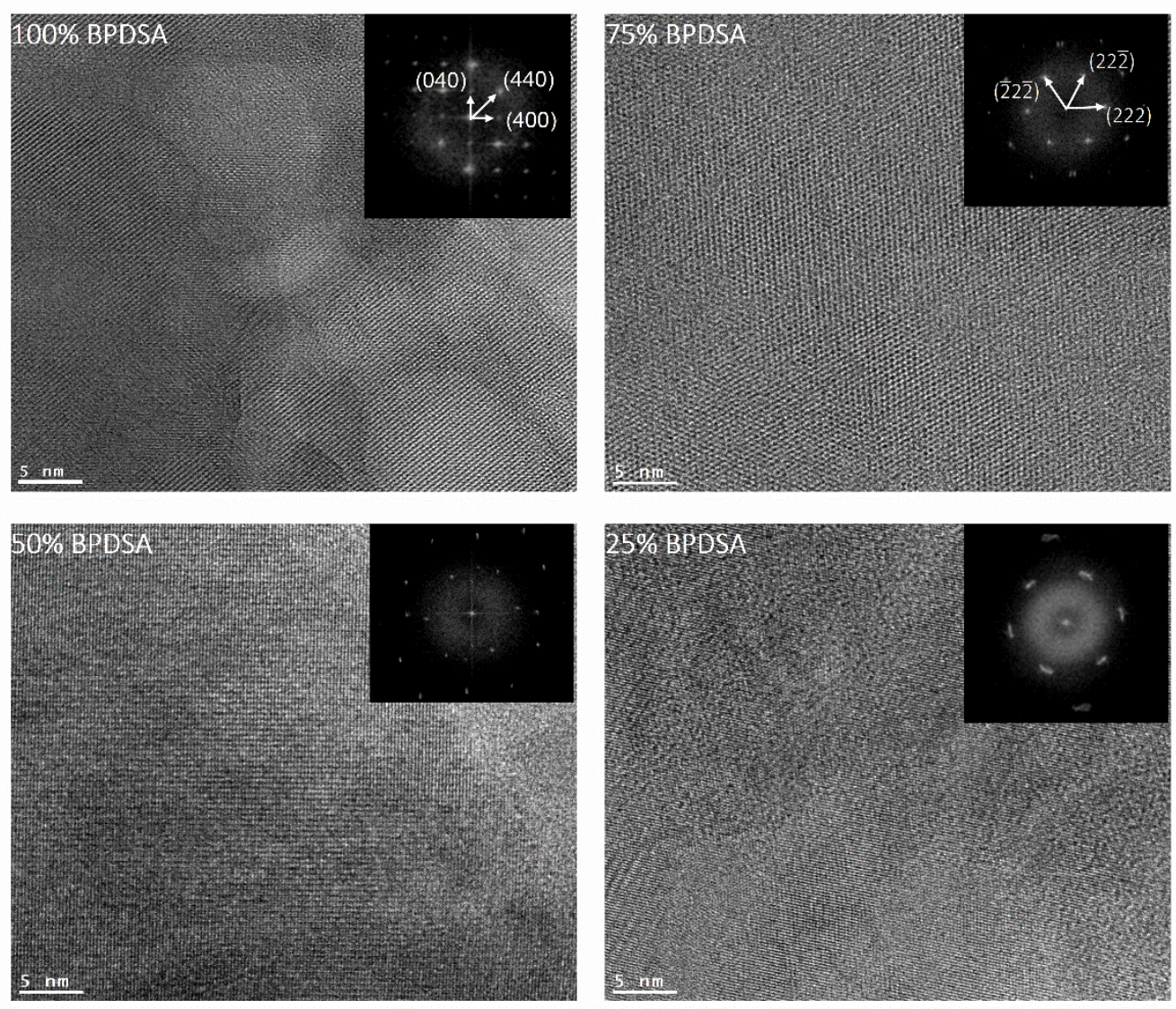

Figure S1. High-resolution transmission electron microscope and processed fast Fourier transform (FFT) images of P(Py:BPDSA:Py) film incorporating varying BPDSA content. The FFT image of P(Py:BPDSA:Py)-100, 75, and 50 revealed that the molecular arrangement of P(Py:BPDSA:Py) corresponded to an FCC crystal structure. ${ }^{1}$ 

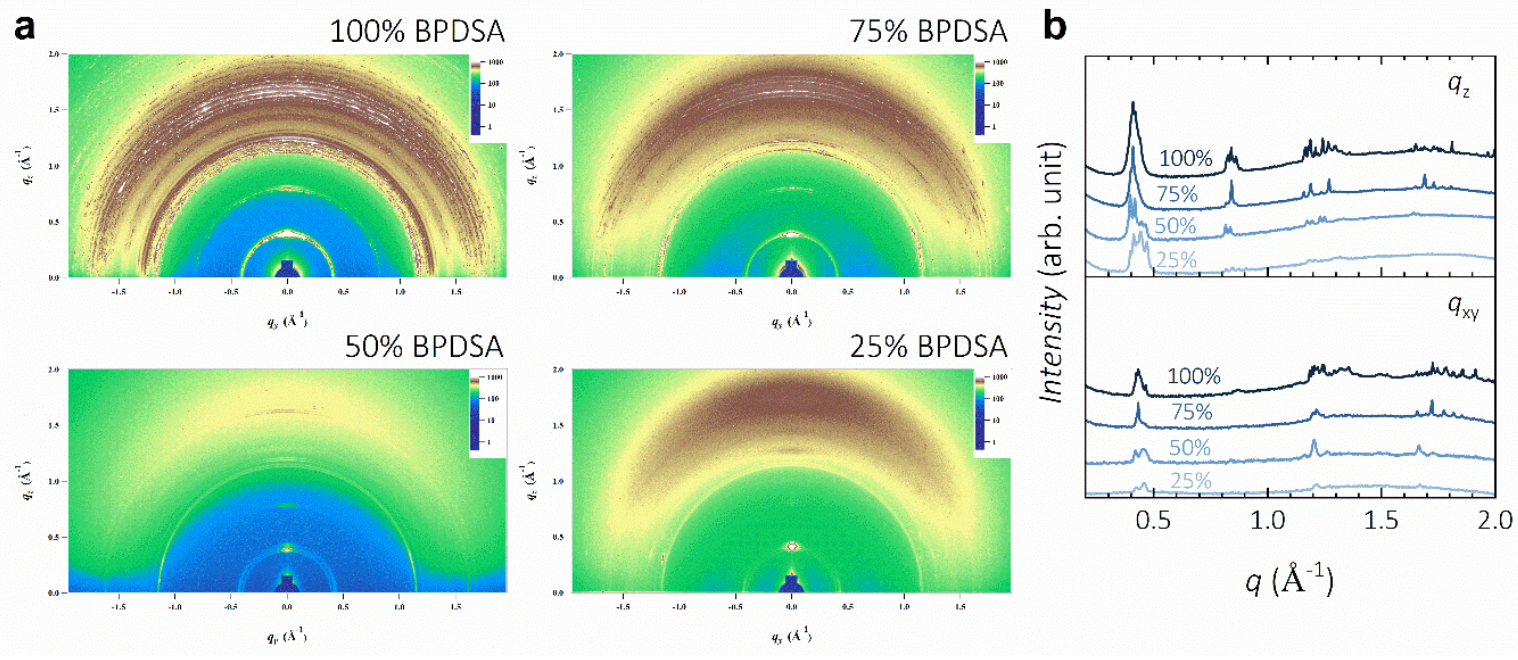

Figure S2. (a) 2D GIWAXD images and (b) vertical (upper panel) and horizontal (lower panel) line-cut profiles of P(Py:BPDSA:Py)-n film incorporating varying BPDSA content. 

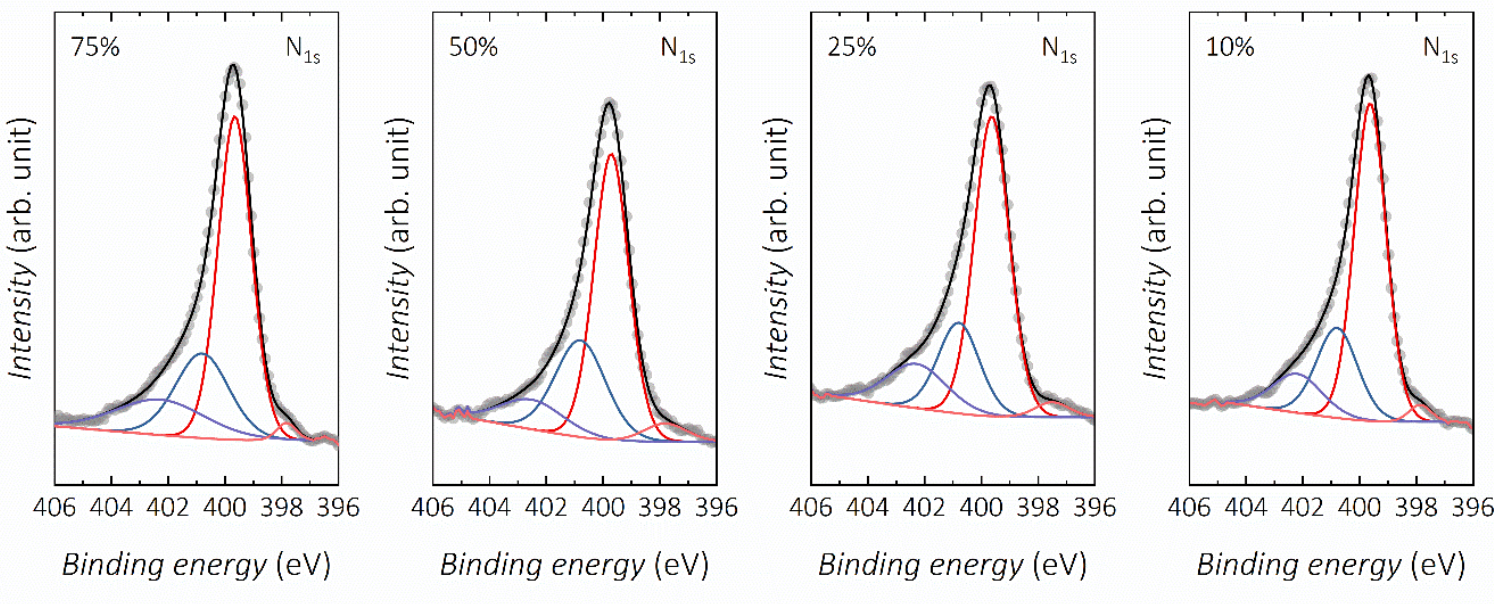

\section{Binding energy (eV)}
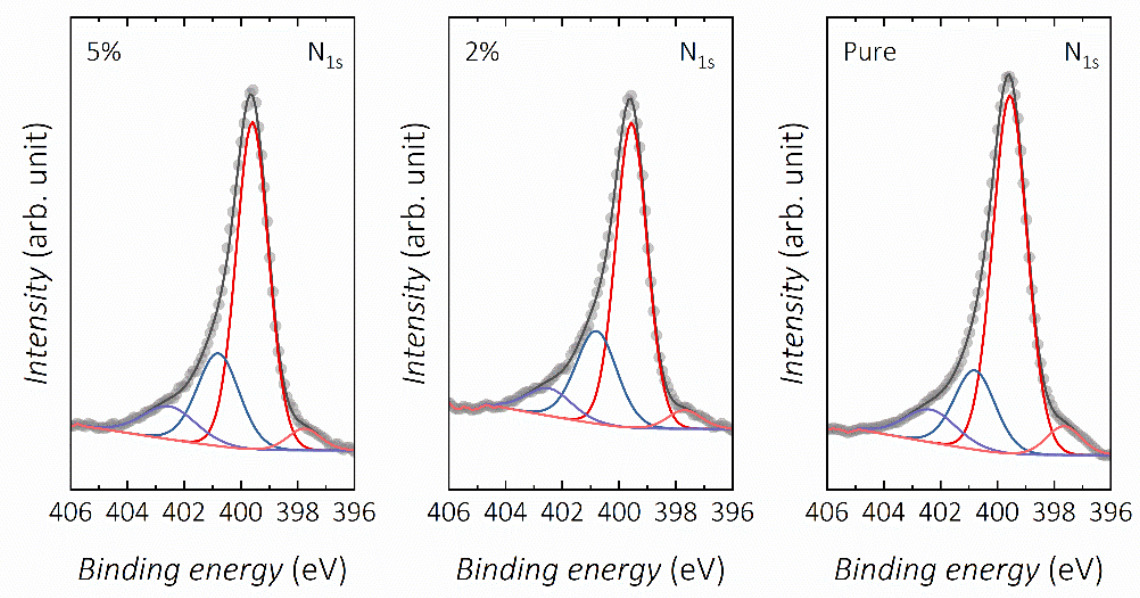

Figure S3. The $\mathrm{N}_{1 \mathrm{~s}}$ signals of $\mathrm{P}(\mathrm{Py}: \mathrm{BPDSA}: \mathrm{Py})$ films incorporating varying BPDSA content and pure polypyrrole film. The deconvoluted peaks indicate the presence of neutral nitrogen (red), $-\mathrm{NH}^{\bullet+}-($ blue $),=\mathrm{NH}^{+}-($purple $)$, and imine $(-\mathrm{NH}=$, pink) states. 
a

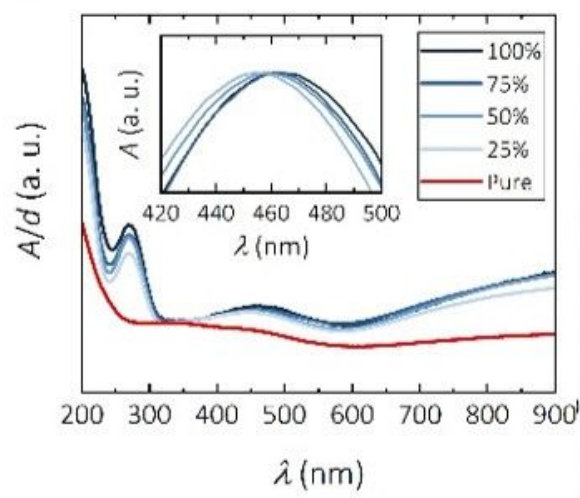

b

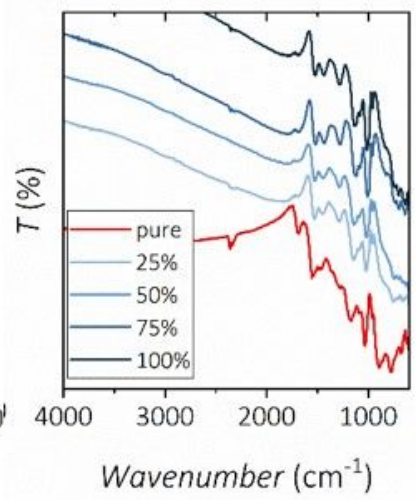

C

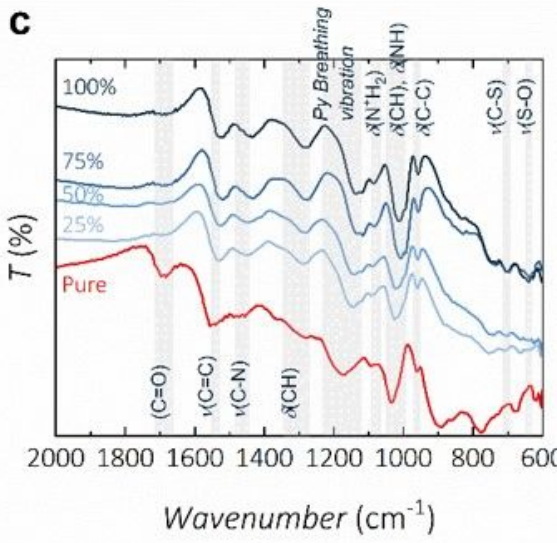

Figure S4. (a) Ultraviolet-visible absorption spectra of P(Py:BPDSA:Py)-n films and pure polypyrrole film with intensities normalized by film thickness. The peak maxima of polaron band obtained from P(Py:BPDSA:Py)-n films were blue-shifted with decreasing BPDSA content (inset). (b) Fourier-transform infrared (FT-IR) spectra of P(Py:BPDSA:Py)-n films and pure polypyrrole film. (c) local magnified FT-IR spectra of P(Py:BPDSA:Py)-n films and pure polypyrrole film. 


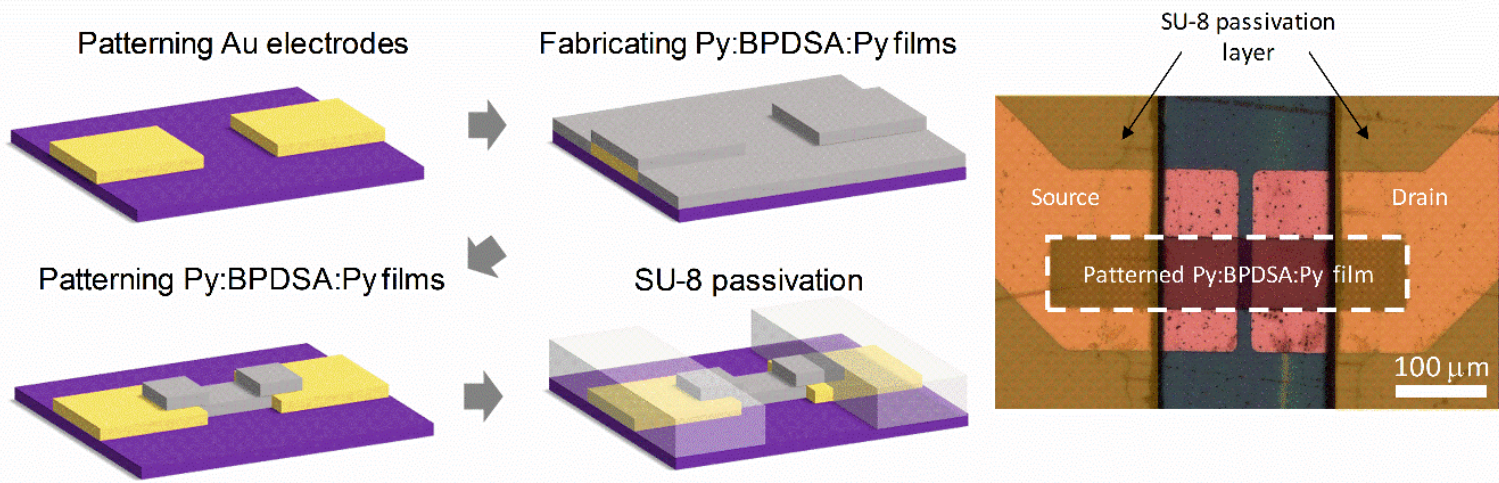

Figure S5. Fabrication process of organic electrochemical transistor using P(Py:BPDSA:Py)$\mathrm{n}$ film as a channel and the optical micrograph of P(Py:BPDSA:Py) OECT after P(Py:BPDSA:Py) patterning and SU-8 passivation. 

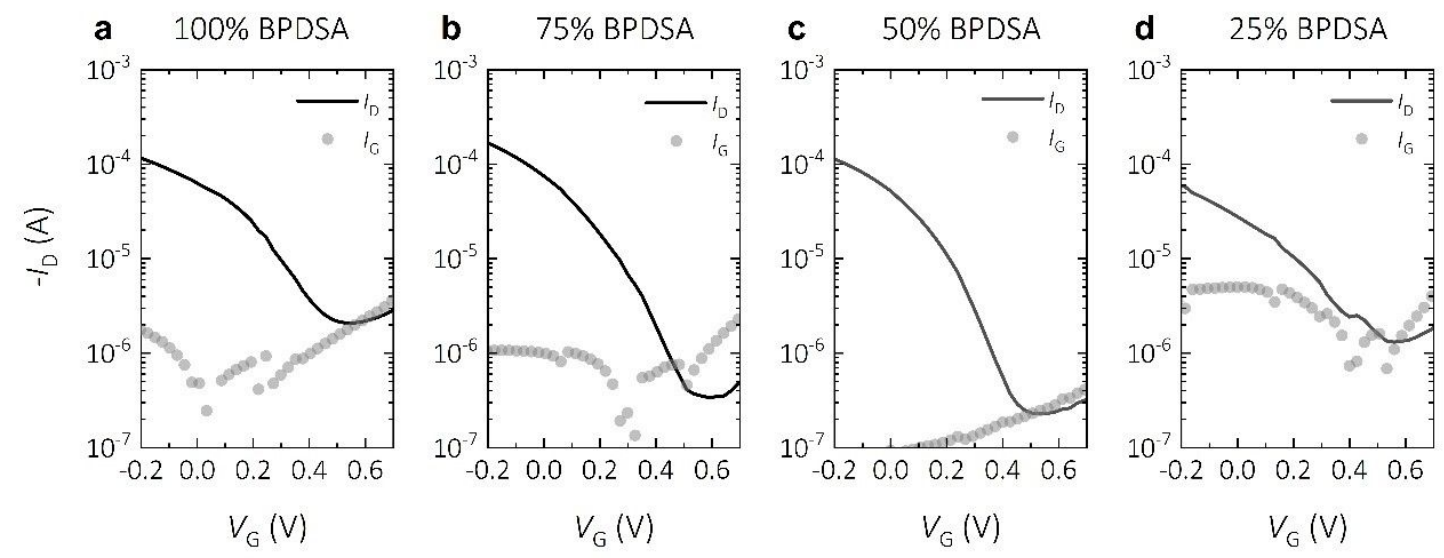

Figure S6. Saturation-regime transfer characteristics of P(Py:BPDSA:Py)-n OECT devices while $V_{\mathrm{D}}$ was fixed at $-0.6 \mathrm{~V}$. 


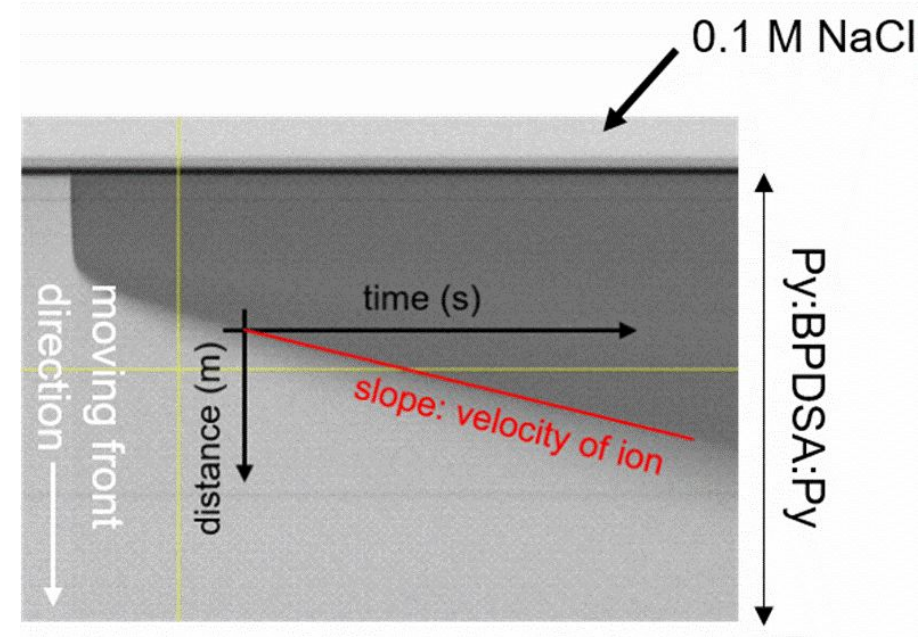

Figure S7. Representative orthogonal view image obtained by processing the movie clip recorded during moving front experiment. A pixel on the x-axis represented $1 \mathrm{~ms}$, so the slope appeared on the image is drift velocity of ion. 


\section{Supplementary References}

[1] J. W. Edington, Practical Electron Microscopy in Materials Science: Monograph 2, Electron Diffraction in the Electron Microscope, MacMillan, 1975. 